\title{
Relacion Entre La Densidad Óptima Agronomica Y El Número De Granos Por Planta En Maíz (Zea Maysl.)
}

\author{
Cecilia Cerliani, Ingeniera Agrónoma. \\ Dr. Gabriel P. Esposito, \\ Dr. Federico D. Morla, \\ Guillermo R. Balboa, MSc. \\ Rafael A. Naville, Ingeniero Agrónomo.
}

Departamento de Producción Vegetal, Facultad de Agronomía y Veterinaria. Universidad Nacional de Río Cuarto, Río Cuarto, Córdoba, Argentina

Doi: 10.19044/esj.2018.v14n9p29 URL:http://dx.doi.org/10.19044/esj.2018.v14n9p29

\begin{abstract}
The density of sowing (D) is one of the main management practices that influences the yield (Y) of corn. There exists a density value in which the yield is maximum (OPD), depending on the environment, the genotype and its interaction. The objectives of this project were: i-To determine the OPD for two corn genotypes in different productive environments; ii- Analyze the relationship between the number of kernel fixed per plant (KNP) and its plant growing rate (PGR) to different environments and genotypes iii- Determine the KNP that is related to the OPD for two corn genotypes. Three experiments were carried out in different locations (L) of Córdoba (Argentina) during 2013/14, comparing 2 genotypes (G) in 2 management zones (MZ). The statistical design was random blocks, with a factorial arrangement of subdivided plots, with L, MZ and G being the primary, secondary and tertiary factors, respectively. In addition, $5 \mathrm{D}$ were planted to obtain the relationships that estimate OPD, PGR, KNP and Y. The results indicate that OPD was affected by L; the relationship between PGR and KNP was not modified by the environment, but by G. The PGR coincident with the OPD was modified by the $\mathrm{G}$ interaction: L. The OPD the PGR was between 2.74 to $4.81 \mathrm{~g} \mathrm{~d}^{-1}$, which were associated with the NGP that varied only between 509 and 603 grains $\mathrm{p}^{-1}$.
\end{abstract}

Keywords: Corn, Plant growth rate (PGR), Optimum plant density (OPD), Yield 


\section{Resumen}

La densidad de siembra (D) es una de las principales prácticas de manejo que influye sobre el rendimiento $(\mathrm{R})$ de maíz. Existe un valor de densidad optima agronómica donde el rendimiento es máximo (DOA), dependiendo del ambiente, del genotipo y su interacción. Los objetivos de este trabajo son: i-Determinar la DOA para dos genotipos de maíz en distintos ambientes productivos; ii- Analizar la relación entre la tasa de crecimiento por planta (TCP) y el número de granos por planta (NGP) para distintos ambientes y genotipos iii- Determinar el NGP que se relaciona con la DOA en dos genotipos de maíz. Se realizaron 3 experimentos en distintas sitio (S) de Córdoba (Argentina) durante la campaña 2013/14, comparando 2 genotipos (G) en 2 zonas de manejo (ZM). El diseño estadístico fue bloques al azar, con un arreglo factorial de parcelas sub-subdivididas, siendo S, ZM y G los factores primario, secundario y terciario, respectivamente. Además, se sembraron $5 \mathrm{D}$ para obtener las relaciones que estimen DOA, TCP, NGP y R. Los resultados mostraron que la DOA fue afectada por $\mathrm{S}$; la relación entre TCP y NGP no fue modificada por el ambiente, pero si por G. A la DOA la TCP se encuentra entre 2,74 a 4,81 $\mathrm{g} \mathrm{d}^{-1}$, que están asociados al NGP que varió solo entre 509 y 603 granos $\mathrm{p}^{-1}$.

Palabras Claves: Maíz, Tasa de crecimiento por planta (TCP), Densidad Optima Agronómica (DOA), Rendimiento

\section{Introducción}

El rendimiento del maíz se incrementó seis veces desde 1939 hasta la actualidad, atribuyéndose un $60 \%$ de este aumento al mejoramiento genético y un $40 \%$ a cambios en las prácticas de manejo. Dentro de estas, se destacan: incremento de la densidad de siembra, aumento en el uso de fertilizantes, mejoras en el control de malezas y diferentes fechas de siembra (Tollenar y Lee, 2011).

Dentro de las prácticas de manejo, la densidad de siembra es un factor de producción muy importante en este cultivo, debido al efecto que tiene sobre la producción y partición de materia seca en la planta, afectando el rendimiento (Andrade et al., 1996).

La respuesta del rendimiento a la densidad de siembra puede describirse en tres fases: a) un incremento lineal a densidades muy bajas b) un incremento parabólico en densidades intermedias y c) una respuesta parabólica negativa en altas densidades (Tollenar, 1992, Andrade et al., 1996, Sarlengue et al., 2007).

Existe por lo tanto un valor de densidad en el cual se produce el máximo rendimiento, dicho valor se conoce como densidad óptima 
agronómica (DOA), y alrededor de este la respuesta del rendimiento a la densidad puede describirse como un polinomio de segundo orden (Sarlengue et al., 2007; Tollenar y Lee, 2011; Hernandez et al., 2014).

La forma cuadrática de la respuesta delrendimiento del maíza la densidad se explica, a diferencia de los restantes cultivos, porque la relación que existe entre el número de granos por planta (NGP) y la tasa de crecimiento individual (TCP) durante el periodo crítico para la definición del rendimiento ( \pm 15 días alrededor de floración), esta relación es del tipo inversa mientras que en otros cultivos es lineal (Andrade et al., 1999).

En condiciones hídricas y nutricionales no limitante, la relación entre NGP y TCP se caracteriza por presentar un valor umbral que indica la mínima TCP necesaria para producir al menos un grano, y otro valor de TCP a partir del cual incrementos en la misma no significan aumentos en el NGP (Andrade et al., 1999).

Esta relación no se modifica por variaciones en la disponibilidad de recursos ya que los efectos directos sobre el número de granos que puede tener un estrés hídrico y/o nutricional, son pequeños o están correlacionados con el efecto del estrés sobre la TCP. De este modo, la relación entre la TCP durante el periodo crítico y el NGP depende exclusivamente del genotipo (Andrade et al., 2002). Sin embrago D'Andrea et al., (2008) encontraron que diferente oferta de nitrógeno en determinados genotipos modificaron esta relación.

Diversos autores han demostrado que es la genética el principal factor que modifica la relación entre TCP y NGP, dado que los híbridos actuales toleran mayor densidad que los disponibles anteriormente, por lo que poseen mayor DOA (Echarte y Andrade, 2003; Tollenar y Lee, 2011; Di Matteo et al., 2016), esta mayor tolerancia a la densidad está asociada a un menor aborto de granos y al menor número de plantas estériles (Echarte y Andrade, 2003), por la capacidad de estos híbridos de fijar granos a inferiores tasas de crecimiento por planta que los híbridos más antiguos (Echarte et al., 2013).

Estas características del maíz determinan que es necesario ajustar la DOA para cada híbrido según la disponibilidad de recursos puesto que en densidades muy altas la competencia intraespecifica hace que disminuya drásticamente la tasa de crecimiento por planta, llegando incluso a valores menores que el valor umbral para la producción de granos, quedando la planta estéril. Por el contrario, si la densidad es muy baja, la tasa de crecimiento individual es alta, pero, debido a limitaciones morfogenéticas del maíz, la falta de plantas no es compensada por una mayor producción individual (Edmeades y Daynard, 1979; Andrade et al., 1999; Sarlengue et al., 2007).

Por otro lado, la disponibilidad de recursos varia espacialmente, tanto a escala regional como a escala de lote (Esposito, 2013), como consecuencia de la variación del clima y de las propiedades físicas y químicas de los suelos. La variabilidad del suelo emerge de complejas interacciones entre factores 
naturales (material parental, topografía, clima y actividad de organismos) y antrópicos o de manejo (labranza, fertilización, aplicación de enmiendas, etc) (Echeverría y García, 2014).

La región suroeste de la provincia de Córdoba (Argentina) se caracteriza por presentar una alta heterogeneidad ambiental, encontrándose distintas aéreas fisiográficas con características de relieve y suelos contrastantes entre sí (Degioanni et al., 2008). Si bien las propiedades del suelo varían a pequeña escala, existen dentro de los lotes zonas con características homogéneas las cuales se denominan “Zonas de Manejo”, y se definen como zonas dentro de un lote que poseen una combinación relativamente homogénea de los factores limitantes del rendimiento y para la cual una única dosificación de insumo es adecuada (Schepers et al., 2004; Vrindts et al., 2005; Moral et al., 2010). Por lo tanto, si bien dentro de las zonas las propiedades son homogéneas y por ende la disponibilidad de recursos es similar, entre zonas existen diferencias en la oferta de recursos.

Al modificar la cantidad de individuos por superficie se modifica la disponibilidad de recursos por planta, así al mejorar la disponibilidad de recursos se debería aumentar la cantidad de individuos para mantener la TCP constante. Esto hace que en ambientes con más recursos soporten una mayor densidad y viceversa (Bragachini et al., 2012; Horbe et al., 2013; Al-Kaisi y Yin 2003). Varios autores han demostrado que la DOA varía al cambiar de ambientes, aun dentro de un mismo lote (Shanahan et al., 2004; Horbe et al., 2013). Estos estudios previos demuestran que la DOA se modifica con las condiciones ambientales y por lo tanto es necesario decidir la densidad en cada condición ambiental. Además, la densidad óptima puede variar entre genotipos aún para similares condiciones ambientales (Sarlengue et al., 2007).

Sin embargo, es poco conocida la relación entre la DOA y la TCP en la cual crecen las plantas a esa densidad. Además, si la relación entre el NGP y el TCP depende solamente del genotipo y si la DOA coincide con una producción individual constante, como producto de esta relación podrían realizarse recomendaciones de densidad de siembra de maíz dependiente del genotipo y de la variabilidad espacial del rendimiento. En tal sentido, los objetivos de este estudio son: i-Determinar la densidad óptima agronómica para dos genotipos de maíz en distintos ambientes productivos; ii- Analizar la relación entre la TCP y el NGP para distintos ambientes y genotipos iiiDeterminar el número de granos por planta que se relaciona con la densidad óptima agronómica para dos genotipos de maíz.

\section{Materiales y métodos}

\section{Descripción de los ensayos y tratamientos.}

Se llevaron a cabo tres experimentos durante la campaña 2013/14 en la región sur de la provincia de Córdoba, Argentina. En la Tabla 1 se presentan 
la localización de los sitios experimentales, Subgrupos de suelo (INTA y Agencia de Córdoba Ambiente, 2003) en cada sitio, fecha de siembra y datos climáticos registrados durante el ciclo del cultivo para cada sitio.

Tabla 1. Localización de los sitios experimentales,subgrupo de suelo, fecha de siembra y datos climáticos durante el ciclo de crecimiento del cultivo.

\begin{tabular}{|c|c|c|c|c|c|}
\hline $\begin{array}{c}\text { Sitio } \\
\text { Experimental }\end{array}$ & Ubicación & $\begin{array}{c}\text { Subgrupo } \\
\text { de Suelo }\end{array}$ & $\begin{array}{l}\text { Fecha de } \\
\text { siembra }\end{array}$ & $\begin{array}{c}\text { Precipitaciones } \\
(\mathrm{mm})\end{array}$ & $\begin{array}{c}\text { Temperatura } \\
\text { media }\left({ }^{\circ} \mathrm{C}\right)\end{array}$ \\
\hline $\begin{array}{c}\text { I. Pueblo } \\
\text { Italiano (PI) }\end{array}$ & $\begin{array}{c}\left(33^{\circ} 52^{\prime} 46,56^{\prime \prime} \mathrm{S} y\right. \\
\left.62^{\circ} 57^{\prime} 36,24 ” O\right)\end{array}$ & $\begin{array}{l}\text { Haplustol } \\
\text { éntico }\end{array}$ & $17 / 10 / 2013$ & 525 & 23 \\
\hline $\begin{array}{c}\text { II. Las } \\
\text { Vertientes (LV) }\end{array}$ & $\begin{array}{c}\left(33^{\circ} 18^{\prime} 56,27^{\prime \prime} \mathrm{S} \text { y }\right. \\
\left.64^{\circ} 34^{\prime} 22,78^{\prime \prime} \mathrm{O}\right)\end{array}$ & $\begin{array}{l}\text { Haplustol } \\
\text { údico }\end{array}$ & $10 / 12 / 2013$ & 401 & 18 \\
\hline $\begin{array}{l}\text { III. Chajan } \\
\text { (Ch) }\end{array}$ & $\begin{array}{c}\left(33^{\circ} 38^{\prime} 24,29^{\prime \prime} \mathrm{S} \text { y }\right. \\
\left.64^{\circ} 58^{\prime} 03,29^{\prime \prime} \mathrm{O}\right)\end{array}$ & $\begin{array}{l}\text { Haplustol } \\
\text { éntico }\end{array}$ & $16 / 12 / 2013$ & 419 & 20 \\
\hline
\end{tabular}

En todos los sitios experimentales el cultivo antecesor fue soja. La siembra se realizó con una distancia entre surcos de 0,52 $\mathrm{m}$. En todos los casos se fertilizó a la siembra con 12, 40 y $10 \mathrm{~kg} \mathrm{ha}^{-1}$ de N, P y S respectivamente. En el estadio fenológico de V6 (Ritchie y Hanway, 1982) se realizó una refertilización con $100 \mathrm{~kg} \mathrm{ha}^{-1}$ de $\mathrm{N}$ con nitrato de amonio calcáreo como fuente. Mediante control químico se mantuvo los ensayos libres de malezas, plagas y enfermedades.

El diseño experimental utilizado fue en bloques completos al azar, con un arreglo factorial de parcelas sub-subdivididas (Di Rienzo et al., 2005). Los factores primario, secundario y terciario fueron el sitio, la zona de manejo y el genotipo, respectivamente. Se sembraron 5 densidades: 40.000, 60.000, $90.000,120.000$ y 150.000 semillas $\mathrm{ha}^{-1}$ para obtener las relaciones que estimen DOA, TCP, NGP y rendimiento.

\section{Delimitación de las zonas de manejo:}

Previo al establecimiento de los ensayos, se realizó una ambientación de los lotes donde se ubicaron los ensayos a fin de delimitar los ambientes de alta y baja producción (AP y BP) en cada sitio experimental.

La delimitación de ambientes se realizó en base a mapas de rendimiento de campañas previas (ya sean de maíz o soja), los cuales fueron previamente analizados con el programa Quantum Gis Wien 2.8.1 (Quantum GIS Development Team, 2011). Dicho procesamiento consistió, en una primera instancia, en la limpieza del mapa de rendimiento del lote completo, esto es, la eliminación de aquellos datos que por diferentes motivos generan una distorsión de los datos recolectados, es decir, que son inconsistentes o incoherentes con la realidad (Bongiovanni et. al, 2006). Luego, se analizó el mapa resultante a través del software "Management Zone Analyst (MZA)" (Mizzou-ARS, 2000) para obtener las zonas de manejo (ZM). 


\section{Mediciones}

Producción de Materia Seca, se determinó en los estadios fenológicos de V13 y R3 (Ritchie y Hanway, 1982), periodo coincidente con el periodo crítico del cultivo de maíz.

La tasa de crecimiento por planta durante el periodo V13-R3, se calculó según la ecuación propuesta por Andrade et al. (1999).

Rendimiento y sus componentes se determinaron mediante cosecha manual al momento de madurez de cosecha. Se tomaron 4 muestras por tratamiento y repetición. En cada muestra se recolectaron todas las espigas que se encontraron en 5 metros lineales de los surcos centrales de cada parcela. Posteriormente se desgranaron mecánicamente y se pesó la muestra.

En ese mismo momento se determinó el peso de granos, para lo cual se pesaron dos muestras de 500 granos cada una por tratamiento y repetición. El número de granos se determinó en base al peso de 1000 granos y el rendimiento según lo propuesto por Andrade et al. (1999).

\section{Relaciones entre variables}

A partir de los datos de rendimiento y densidad de plantasse ajustó un modelo de respuesta del rendimiento a la densidad de tipo cuadrático (Andrade et al. 1996; Sarlengue et al., 2007), para cada genotipo en cada sitio experimental, zona de manejo y bloque.

La densidad óptima agronómica (DOA), se determinó para cada híbrido en cada repetición, zona de manejo y para cada sitio experimental como la primera derivada de la función de rendimiento del maíz con relación a la densidad de plantas y posteriormente se igualo a 0 y se despejo densidad (Sarlengue et al., 2007).

La relación entre NGP y TCP, se estimó mediante el ajuste de la función propuesta por Andrade et al.,(1999):

$$
N G P=a-\left({ }^{b} / T C P\right) \text { [Ecuación I] }
$$

donde a y b son parámetros determinados mediante regresión no lineal. NGP corresponde a los números de granos por plantas; TCP a la tasa de crecimiento por planta en el período crítico de definición del rendimiento para maíz.

Esta función se obtuvo para cada genotipo en los distintos sitios experimentales y zonas de manejo y se compraron con una única función para cada genotipo obtenida a partir de todo el set de datos.

Por último, para determinar el NGP asociado con la DOA, se estimó la relación entre la tasa de crecimiento y densidad mediante el ajuste de una función exponencial (Ecuación II), para cada genotipo en cada sitio experimental y zona de manejo.

$$
\triangle T C P\left(g d \rrbracket^{-1}\right)=a E_{x p}^{-b D} \quad \text { [Ecuación II] }
$$


donde TCP es la tasa de crecimiento por planta $\left(\mathrm{g} \mathrm{d}^{-1}\right)$; a y b son parámetros del modelo; D es la densidad de plantas a cosecha.

A partir de la densidad óptima agronómica obtenida y la ecuación II se obtuvo el valor de TCP correspondiente con la DOA.Posteriormente a través de la Ecuación I y el valor de TCP se estimó el número de granos por planta correspondiente con la DOA.

Todas las relaciones fueron ajustadas y comparadas entre sí mediante el programa estadístico GraphPad Prism version 5.00 para Windows, (GraphPad Software, San Diego California USA). Además, se realizó un ANAVA y test de comparación de medias LSD de Fisher $(\alpha=0.05)$, mediante el software estadístico INFOSTAT versión 2012 (Di Reinzo et al., 2016).

\section{Resultados y discusión}

\section{Relación entre rendimiento y densidad de plantas.}

La respuesta del rendimiento a la densidad de plantas fue de tipo cuadrática para ambos genotipos y varió entre estos y los sitios evaluados (sitio y zona de manejo) (Figura 1).

En PI y LV se encontró que en la zona AP el rendimiento fue superior en todas las densidades al obtenido en la zona BP, destacándose que la mayor diferencia entre las zonas se encontró en LV. En Ch la diferencia entre zonas solo fue estadísticamente significativa para el híbrido DK 692 VT3P, mientras que para el híbrido DK 7210 VT3P las curvas de respuesta no difirieron entre los ambientes delimitados previamente.

Al cambiar de ambientes, ya sea por cambio de sitio y/o zona, se altera la oferta de recursos (radiación, agua (Tabla 1), etc), como así también varían las propiedades físicas y químicas de los suelos (Bongiovanni et al., 2006; Esposito, 2013).Esta variación en la respuesta del rendimiento de maíz a la densidad de plantas entre sitioes y zonas coincide con los resultados encontrados por otros autores quienes hallaron que esta relación cambia con el ambiente (Bullock et al., 1998; Shanahan et al. 2004; Bragachini et al. 2012; Horbe et al., 2013; Martinez Bolognaet al., 2014; Cerliani et al., 2014). 

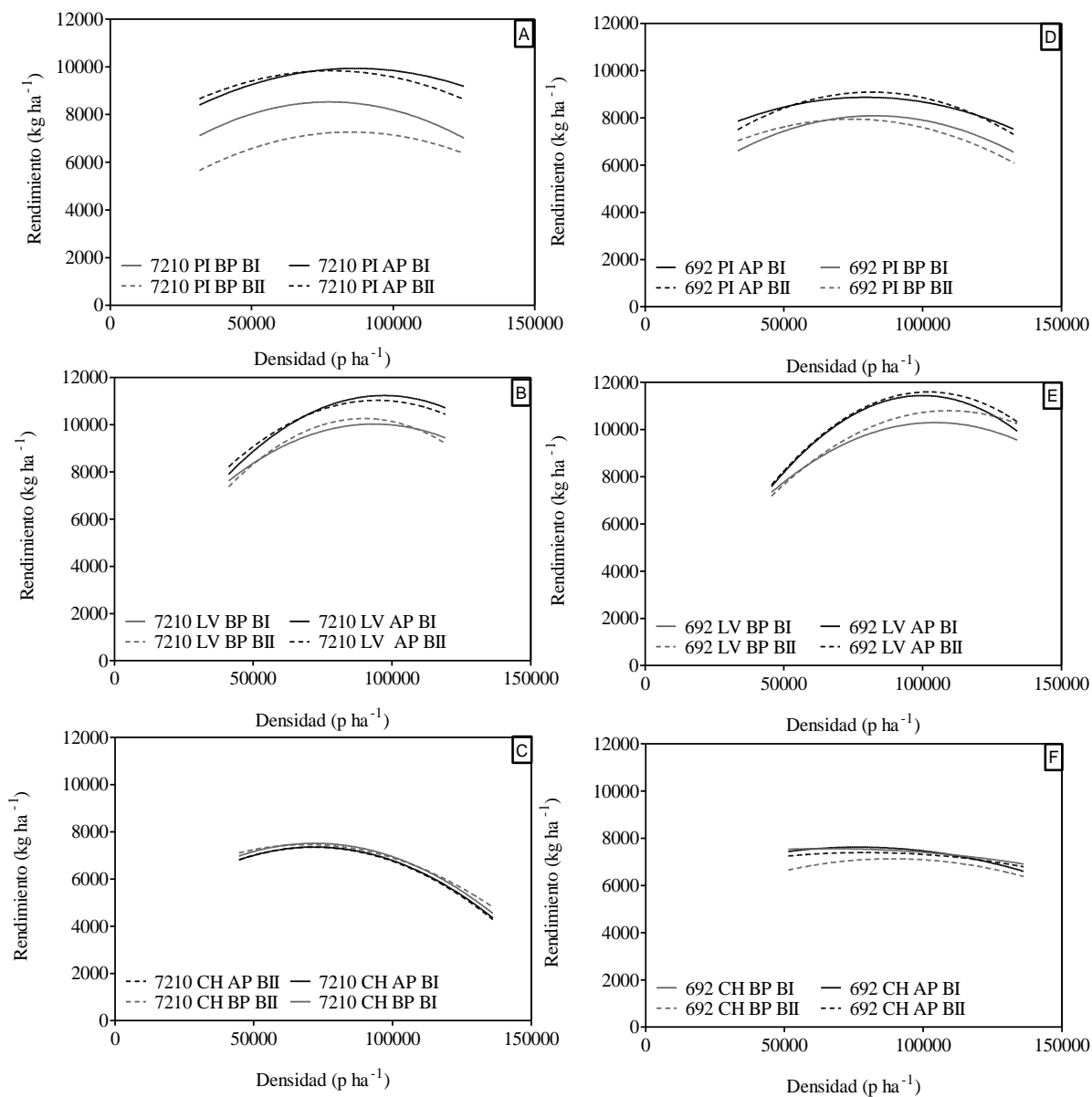

Figura 1. Relación entre Rendimiento $\left(\mathrm{kg} \mathrm{ha}^{-1}\right)$ y densidad de plantas $\left(\mathrm{p} \mathrm{ha}^{-1}\right)$ para el DK 7210 VT3P en Pueblo Italiano (A), Las Vertientes (B), Chajan (C) y para el DK 692 VT3P en Pueblo Italiano (D), Las Vertientes (E), Chajan (F) para ambas zonas de manejo.

\section{Relación entre tasa de crecimiento por planta y numero de granos por planta}

Los datos de ambos híbridos ajustaron a una única ecuación de tipo inversa $(\mathrm{NG}=\mathrm{a}-(\mathrm{b} / \mathrm{TCP})$, que no difirió estadísticamente el ajuste individual de cada set de datos definido por sitio y zona, $\mathrm{F}=7,19 ; \mathrm{p}<0,0001$ y $\mathrm{F}=6,68$; p<0,0001 para DK 692 VT3P; y DK 7210 VT3P, respectivamente (Tabla 2).

Tabla 2. Ecuación de ajuste de regresión inversa entre números de granos por planta y tasa de crecimiento por planta durante el periodo crítico en cada sitio y ambiente para ambos híbridos (DK 7210VT3P y DK 692VT3P) y ecuación general para cada híbrido.

\begin{tabular}{cccccc}
\hline Sitio & Zona & Ecuación & R2 & Parámetro A & Parámetro B \\
\hline DK 7210 VT3P & NG $=\mathbf{7 2 9 , 2}-\left(\mathbf{6 8 1}, \mathbf{7} \mathbf{T C P}^{-1}\right)$ & $\mathbf{0 , 4 8}$ & & \\
\hline PI & AP & NG $=751,2-\left(941,6 \mathrm{TCP}^{-1}\right)$ & 0,55 & 0,6094 & 0,0737
\end{tabular}




\begin{tabular}{cccccc} 
& $\mathrm{BP}$ & $\mathrm{NG}=704,8-\left(719,5 \mathrm{TCP}^{-1}\right)$ & 0,56 & 0,5494 & 0,7195 \\
\hline \multirow{2}{*}{$\mathrm{LV}$} & $\mathrm{AP}$ & $\mathrm{NG}=792,9-\left(563,1 \mathrm{TCP}^{-1}\right)$ & 0,55 & 0,0593 & 0,1799 \\
& $\mathrm{BP}$ & $\mathrm{NG}=780,5-\left(732,4 \mathrm{TCP}^{-1}\right)$ & 0,58 & 0,1457 & 0,6469 \\
\hline \multirow{2}{*}{$\mathrm{CH}$} & $\mathrm{AP}$ & $\mathrm{NG}=709,8-\left(604,4 \mathrm{TCP}^{-1}\right)$ & 0,65 & 0,5102 & 0,303 \\
& $\mathrm{BP}$ & $\mathrm{NG}=711,2-\left(772,3 \mathrm{TCP}^{-1}\right)$ & 0,52 & 0,6651 & 0,448 \\
\hline \multirow{2}{*}{ DK 692 VT3P } & $\mathrm{NG}=\mathbf{7 6 9 , 6}-\left(\mathbf{7 1 1 , 7} \mathbf{T C P}^{-1}\right)$ & $\mathbf{0 , 3 7}$ & & \\
\hline \multirow{2}{*}{$\mathrm{PI}$} & $\mathrm{AP}$ & $\mathrm{NG}=799,8-\left(1069 \mathrm{TCP}^{-1}\right)$ & 0,36 & 0,6731 & 0,148 \\
& $\mathrm{BP}$ & $\mathrm{NG}=772,2-\left(945,9 \mathrm{TCP}^{-1}\right)$ & 0,47 & 0,9666 & 0,6282 \\
\hline \multirow{2}{*}{$\mathrm{LV}$} & $\mathrm{AP}$ & $\mathrm{NG}=834,4-\left(610 \mathrm{TCP}^{-1}\right)$ & 0,63 & 0,756 & 0,2311 \\
& $\mathrm{BP}$ & $\mathrm{NG}=865,4-\left(685,2 \mathrm{TCP}^{-1}\right)$ & 0,5 & 0,0748 & 0,8405 \\
\hline \multirow{2}{*}{$\mathrm{CH}$} & $\mathrm{AP}$ & $\mathrm{NG}=764,3-\left(744,5 \mathrm{TCP}^{-1}\right)$ & 0,63 & 0,8969 & 0,7233 \\
& $\mathrm{BP}$ & $\mathrm{NG}=780,7-\left(778,5 \mathrm{TCP}^{-1}\right)$ & 0,45 & 0,8318 & 0,1522 \\
\hline
\end{tabular}

Los parámetros de este ajuste fueron para el DK 7210VT3P $a=729,2 \pm 16,72$ y $b=681,7 \pm 47,10$, con un $\mathrm{R}^{2}$ de 0,48 y para el DK 692 VT3P $a=769,6 \pm 24,33$ y $b=711,7 \pm 62,70, \quad$ con un $\mathrm{R}^{2}$ de 0,37 ; difiriendo estadísticamente entre ellos ( $\mathrm{p}=0,0377$ ) (Figura 2).

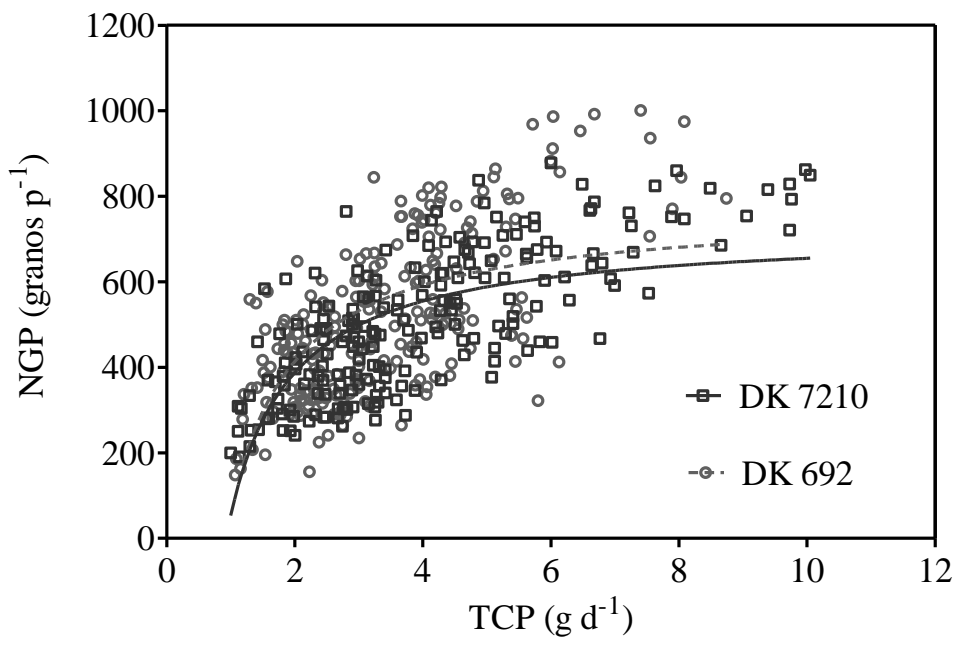

Figura 2. Relación entre el número de granos por planta (granos $\left.\mathrm{p}^{-1}\right)$ y tasa de crecimiento por planta durante el periodo crítico $\left(\mathrm{g} \mathrm{d}^{-1}\right)$, para el híbrido DK 692 VT3P (línea punteada) y DK 7210 VT3P (línea llena) en todos los sitios y ambientes evaluados.

En la Figura 2 puede observarse que ambos genotipos se ajustaron a una regresión inversa, con parámetros $a$ y $b$ que están dentro del rango de valores encontrados por Andrade et al. (2002) quien determinó esta relación para distintas condiciones de disponibilidad de agua, nitrógeno y radiación. 
En este estudio se encontraron diferencias entre genotipos en la relación entre el NGP y la TCP. Esto coincide con lo encontrado en la bibliografía, donde se señala que esta curva cambia entre híbridos antiguos y modernos (Echarte et al., 2013) y entre líneas e híbridos (F1) resultantes de su cruzamiento (Echarte y Tollenar, 2006).

Para los genotipos evaluados se encontró un valor umbral de TCP por debajo del cual la planta no produce granos de 0,93 y $0,92 \mathrm{~g} \mathrm{~d}^{-1}$, para DK 7210 VT3P y DK 692 VT3P, respectivamente. Valores levemente inferiores a los reportados por Andrade et al. (1999) quienes encontraron un umbral de $1 \mathrm{~g} \mathrm{~d}^{-}$ 1 para el híbrido DK 636 (genotipo de la década de 1990) al igual que el umbral encontrado por Tollenar et al. (1992). Sin embargo, fueron similares al rango de valores umbrales de TCP descriptos por Echarte y Tollenar (2006) que varió de 0,67 a 1,2 $\mathrm{g} \mathrm{d}^{-1}$ para dos híbridos evaluados durante 2003 y 2004 (adaptado ya que esto está en GDD).

El menor umbral de la TCP necesaria para producir granos, encontrado en este trabajo podría estar asociado al mejoramiento genético, el cual condujo a que en híbridos más modernos sea inferior, debido a la selección para tolerar altas densidades (Tollenar y Lee, 2011).

Si bien en bajas tasas de crecimiento ambos híbridos se comportan de manera similar, conforme aumenta la TCP el híbrido DK 692 VT3P posee más granos para una misma tasa, lo cual indica una mayor potencialidad en la producción de granos. Así, cuando la TCP fue de $4 \mathrm{~g} \mathrm{~d}^{-1}$ el NGP fue de $593 \mathrm{y}$ 560 granos $\mathrm{p}^{-1}$, para el DK 692 VT3P y DK 7210 VT3P, respectivamente.

Coincidentemente con lo planteado por otros autores, la relación entre el número de granos por planta y la tasa de crecimiento por planta fue genética dependiente (Echarte y Tollenar 2006; Echarte et al., 2013), variando entre genotipos distintos, pero no se modificó al cambiar de ambiente. Según Andrade et al. (2002) modificaciones en la disponibilidad de agua o en la disponibilidad de nitrógeno no generan cambios en la relación entre el número de granos y la tasa de crecimiento con respecto al mismo híbrido en condiciones no limitantes de agua y nutrientes. En contraposición, D’Andrea et al. (2008), indicaron que al variar la oferta de nitrógeno la relación entre el número de granos y la TCP fue modificada y esta variación en la relación era dependiente del genotipo.

\section{Relación entre densidad y tasa de crecimiento por planta}

En ambos genotipos se encontró que la TCP disminuyó de manera exponencial al incrementar la densidad de plantas, y que esta relación entre ambas variables difirió estadísticamente entre los ambientes y los genotipos (Figura 3). 

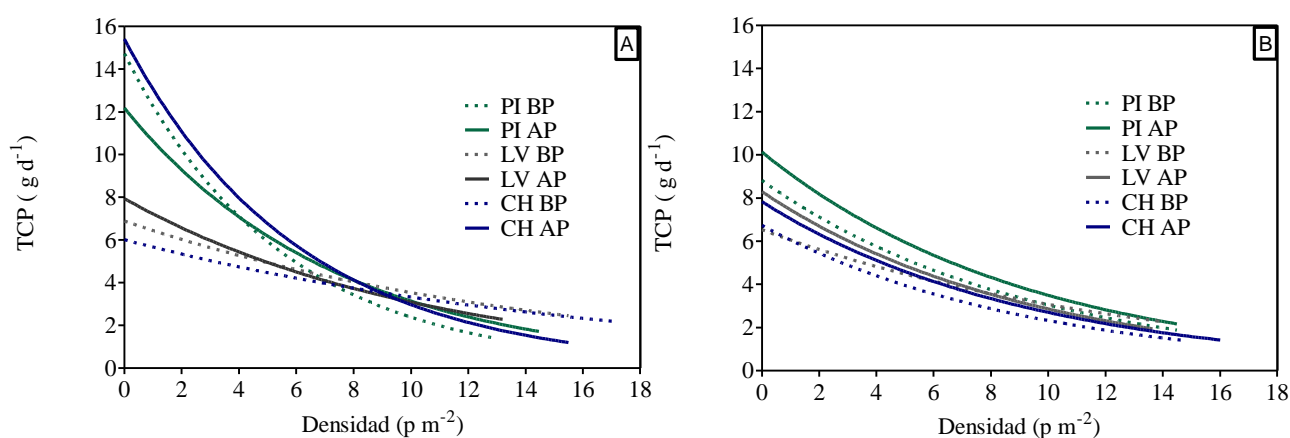

Figura 3. Relación entre TCP durante el periodo crítico $\left(\mathrm{g} \mathrm{d}^{-1}\right)$ y densidad de plantas $\left(\mathrm{p} \mathrm{m}^{-2}\right)$, para el híbrido DK 7210 VT3P (a) y para DK 692 VT3P (b) en todos los sitios experimentales y zonas de manejo.

Los resultados encontrados indican que, para una misma densidad, al modificar el ambiente la TCP se modifica, por lo tanto, al variar la zona de manejo y/o sitio la oferta de recursos cambia. Esto podría deberse a cambios en la oferta de radiación, temperatura y agua entre los tres sitios (PI, LV, Ch) (Tabla 1) y además debe considerarse que la disponibilidad de recursos varía espacialmente, como consecuencia de la variación de las propiedades físicas y químicas de los suelos (Espósito, 2013). Por lo tanto, en cada ambiente la respuesta en la disminución de TCP al aumentar la densidad fue diferente.

Por otro lado, se encontró que la relación entre la tasa de crecimiento y la densidad de plantas, no solo se modificó al cambiar de ambiente, sino que también entre genotipo, esto se explicaría por la manera diferencial de los genotipos de captar los recursos y convertirlos en fotoasimilados (Lee y Tollenar, 2007; Echarte et al., 2013; Nagore et al., 2014) ya que la TCP depende de estos procesos.

Estudios similares realizados en Balcarce (Argentina) indicaron que aumentos en la densidad de plantas generaron disminuciones en la biomasa total de la planta y que la respuesta fue diferente entre distintos híbridos evaluados (Sarlengue et al., 2007).

Densidad óptima agronómica, tasa de crecimiento por planta, numero de granos y rendimiento correspondientes con la densidad óptima agronómica

La DOA solo varió significativamente entre los tres sitios evaluados $(p=0,0013)$. LV fue el sitio donde se registraron los mayores valores de DOA (98903 plantas ha ${ }^{-1}$ ), siendo un 33 y $22 \%$ superior a PI y Ch, donde la DOA fue inferior (81271 y 74472 plantas ha $^{-1}$, respectivamente) (Tabla 3).

En el caso del número de granos planta, la tendencia fue la misma, pero en sentido inverso, es decir, LV presentó un menor NGP (515) el cual fue 
estadísticamente inferior al NGP encontrado en Ch y PI (570 y 582, respectivamente) (Tabla 3).

En síntesis, estas dos variables se comportaron de manera inversa, es decir, en el sitio donde mayor fue la DOA, el NGP fue menor y viceversa.

En cuanto al rendimiento que se corresponde con la DOA, varió tanto para el sitio $(p<0,0001)$ evaluado como para las zonas dentro de cada sitio $(\mathrm{p}=0,0006)$. El sitio más productivo fue LV con $10839 \mathrm{~kg} \mathrm{ha}^{-1}$, seguido por PI $8727 \mathrm{~kg} \mathrm{ha}^{-1}$ y en tercer lugar se ubicó Ch $7423 \mathrm{~kg} \mathrm{ha}^{-1}$. En cuanto a las diferencias entre las zonas, la misma fue de un 9 y $19 \%$ superior en AP respecto a $\mathrm{BP}$ en LV y PI, mientras que en $\mathrm{Ch}$ no hubo diferencias entre las zonas delimitadas.

Tabla 3. Densidad óptima agronómica (DOA), tasas de crecimiento por planta (TCP), número de granos (NGP) y rendimiento de maíz correspondientes a la densidad óptima agronómica (RTO) en cada sitio y ambiente para ambos híbridos evaluados (DK 7210 VT3P y DK 692 VT3P).

\begin{tabular}{|c|c|c|c|c|c|c|}
\hline Híbrido & Sitio & Zona & DOA & $\mathrm{TCP}$ & NGP & RTO \\
\hline \multirow[t]{6}{*}{ DK 7210 VT3P } & \multirow{2}{*}{ PI } & $\mathrm{AP}$ & 82297 & 4,09 & 594.84 & 9884 \\
\hline & & $\mathrm{BP}$ & 81276 & 3,42 & 560.29 & 7896 \\
\hline & \multirow{2}{*}{ LV } & $\mathrm{AP}$ & 95903 & 3,07 & 507.00 & 11153 \\
\hline & & $\mathrm{BP}$ & 91727 & 3,34 & 525.33 & 10142 \\
\hline & \multirow{2}{*}{$\mathrm{CH}$} & $\mathrm{AP}$ & 71872 & 4,81 & 587.36 & 7351 \\
\hline & & $\mathrm{BP}$ & 70616 & 4,07 & 571.93 & 7487 \\
\hline \multirow[t]{6}{*}{ DK 692 VT3P } & \multirow{2}{*}{ PI } & $\mathrm{AP}$ & 82909 & 4,29 & 603.62 & 9110 \\
\hline & & $\mathrm{BP}$ & 78604 & 3,59 & 570.91 & 8018 \\
\hline & \multirow{2}{*}{ LV } & AP & 101074 & 2,74 & 509.89 & 11512 \\
\hline & & $\mathrm{BP}$ & 106909 & 2,84 & 518.91 & 10548 \\
\hline & \multirow{2}{*}{$\mathrm{CH}$} & $\mathrm{AP}$ & 77967 & 3,40 & 560.91 & 7509 \\
\hline & & $\mathrm{BP}$ & 77430 & 3,64 & 571.93 & 7344 \\
\hline Híbrido & & & NS & $*$ & NS & NS \\
\hline Sitio & & & $* *$ & $* *$ & $* *$ & $* * *$ \\
\hline Zona & & & NS & NS & NS & $* *$ \\
\hline Hibrido: Sitio & & & NS & $*$ & NS & NS \\
\hline Hibrido: Zona & & & NS & NS & NS & NS \\
\hline Sitio : Zona : Hibrido & & & NS & NS & NS & NS \\
\hline $\mathrm{R} 2$ & & & 0,91 & 0,94 & 0,94 & 0,99 \\
\hline $\mathrm{CV}$ & & & 8,49 & 8,30 & 2,88 & 3,14 \\
\hline
\end{tabular}


En cuanto a la TCP que se relacionó con la DOA, la misma varió en un rango desde 2,74 a 4,81 $\mathrm{g} \mathrm{d}^{-1} \mathrm{y}$ fue afectada por la interacción entre el sitio y el híbrido. Esta interacción indicó que en LV y Ch el DK 7210 VT3P presentó mayores valores de TCP, en tanto que en PI no se encontraron diferencias estadísticas entre la TCP de los híbridos evaluados. Esto podría deberse a que en PI, por la fecha de siembra, el periodo crítico estuvo expuesto a mayores temperaturas, ya que el mismo ocurrió durante finales de diciembre y principios de enero y según lo señalado por Andrade et al. (1999) al exponer el cultivo a altas temperaturas nocturnas durante el periodo crítico, se disminuye su duración y por ende el número de granos fijados disminuye en comparación con un cultivo que crece bajo las mismas condiciones pero con temperaturas nocturnas más frescas. La diferencia en este efecto entre ambos híbridos evaluados en este trabajo podría estar dada por diferencias en las temperaturas cardinales de desarrollo entre ambos, lo cual haría que el DK 7210 VT3P haya sido afectado y el otro genotipo no.

Hernández et al. (2014), encontraron para distintos genotipos evaluados en dos años, la misma relación entre la DOA y el rendimiento, en su estudio las DOA encontradas para los distintos genotipos variaron entre 7,3 y $11,4 \mathrm{p} \mathrm{m}^{-2}$ y los rendimientos fueron desde $10,4 \mathrm{a} 13,5 \mathrm{Mg} \mathrm{ha}^{-1}$. Por su parte Sarlengue et al. (2007) encontraron valores de DOA de 9,9 a $16,7 \mathrm{p} \mathrm{m}^{-2}$, para distintos genotipos, encontrando valores superiores para genotipos de ciclo más corto, que poseen plantas de menor tamaño, respectos a genotipos de ciclo largo los cuales poseen menor DOA y las plantas tienen mayor tamaño. En otro estudio realizado en Argentina, Di Matteo et al. (2016) evaluando híbridos liberados en distintas décadas encontró que la DOA varió entre 9,7 y $16,4 \mathrm{p} \mathrm{m}^{-2}$.

La DOA se modificó al cambiar la oferta de recursos entre los diferentes ambientes, estos resultados son coincidentes con lo encontrado por Hernandez et al., (2014), quienes hallaron diferencias en la DOA entre distintos genotipos y años de evaluación los cuales generaron distintos ambientes productivos en los distintos años de estudio.

En concordancia con esto Horbe et al. (2013), en Brasil encontraron que la DOA varió entre zonas de manejo dentro de un mismo lote, resultados similares fueron encontrados por otros autores en EEUU (Bullock et al., 1998; Shanahan et al., 2004) y a nivel local (Bragachini et al. 2010; Cerliani et al., 2014). En todos estos estudios los ambientes con mayor disponibilidad de recursos fueron los que presentaron una mayor DOA. Esto podría explicarse por el hecho de que en los ambientes de menor calidad (o sea, menor disponibilidad de recursos, como agua y/o nutrientes), aumentos en la población de plantas generan una alta competencia intraespecifica (Bragachini et al., 2010; Horbe et al., 2013). 
Se encontró una relación lineal negativa entre DOA y $\mathrm{TCP}\left(\mathrm{R}^{2}=0,72 \mathrm{y}\right.$ 0,78, para DK 7210 VT3P y DK 692 VT3P, respectivamente), y para la relación DOA y NGP $\left(\mathrm{R}^{2}=0,62\right.$ y 0,70, para DK 7210 VT3P y DK 692 VT3P, respectivamente). Mientras que la TCP y el NGP, presentaron una relación lineal positiva $\left(\mathrm{R}^{2}=0,72\right.$ y 0,98, para DK 7210 VT3P y DK 692 VT3P, respectivamente). Además, se encontró una relación lineal positiva entre el rendimiento y la DOA, para ambos genotipos $\left(\mathrm{R}^{2}=0,73\right.$ y 0,69 , para $\mathrm{DK} 7210$ VT3P y DK 692 VT3P) coincidiendo con lo hallado por Hernández et al. (2014) y Di Matteo et al. (2016).

Estas relaciones, indicarían que existe una compensación dentro del rango de TCP correspondientes con la DOA en el cultivo, según la cual a bajas densidades hay una alta TCP y un alto NGP; a altas DOA la TCP es menor, con menor NGP, pero mayor rendimiento debido a la mayor densidad. Indicando que dentro del rango de densidades óptimas la relación entre TCP y NGP es lineal, esto coincide con lo indicado por Kiniry et al. (1997), quienes proponen que una regresión lineal podría ser usada exitosamente cuando los valores de TCP estén dentro de lo normal.

\section{Conclusión}

La densidad optima agronómica no presentó diferencias entre los genotipos analizados y las zonas de manejo evaluadas en este trabajo, solo varío entre los distintos sitios presentando valores entre 70.616 y 106.909 plantas $\mathrm{ha}^{-1}$.

La relación entre la TCP y el NGP difirió entre genotipos, pero no fue modificada por el ambiente, este último factor generó solo cambios en la TCP.

Para los dos genotipos evaluados, se encontró que el rango de TCP en los cuales crecen las plantas que están en su densidad óptima (DOA) fue de 2,74 a 4,81 $\mathrm{g} \mathrm{d}^{-1}$, y que el NGP en la DOA varió entre 509 y 603 granos $\mathrm{p}^{-1}$.

Debido a la menor variabilidad del peso de los granos, estos resultados permitirían generar prescripciones de densidad de siembra a partir de un rendimiento esperado en cada sector de un lote, ya que es posible obtener la producción por planta (a partir del NGP y el peso de estos). Por lo tanto, de la división entre el rendimiento esperado y la producción por planta podría obtenerse la densidad óptima de plantas a lograr.

\section{References:}

1. Andrade, F.H., Cirilo, A., Uhart, S., \& Otegui, M. (1996). Ecofisiología del Cultivo de Maíz. Editorial La Barrosa. EEA Balcarce, CERBAS, INTA-FCA, UNMP (Eds.). Dekalb Press. Buenos Aires. 292 pp 
2. Andrade, F. H., Vega, C., Uhart, S., Cirilo, A., Cantarero, M., \& Valentinuz, O. (1999). Kernel number determination in maize. Crop Science, 39(2), 453-459.

3. Andrade, F. H., Echarte, L., Rizzalli, R., Della Maggiora, A., \& Casanovas, M. (2002). Kernel number prediction in maize under nitrogen or water stress. Crop Science, 42(4), 1173-1179.

4. Al-Kaisi, M. M., \& Yin, X. (2003). Effects of nitrogen rate, irrigation rate, and plant population on corn yield and water use efficiency. Agronomy journal, 95(6), 1475-1482.

5. Bongiovanni, R., Mantovani, E., Best, S., \& Roel, Á. (2006). Agricultura de precisión: integrando conocimientos para una agricultura moderna y sustentable. Procisur/IICA.

6. Bragachini, M., Mendez, A., Scaramuzza, F., Velez, J. P., \& Vilarroel, D. (2010). Dosificacion variable de insumos. 9no Curso Internacional de Agricultura de Precisión y 4ta Expo de Máquinas Precisas, Córdoba-Argentina.

7. Bragachini, M., Méndez, A., Scaramuzza, F., Villarroel, D. \& Vélez, J.P. (2012) Ensayo de larga duración de manejo de insumos en forma variable. INTA. En: http://inta.gob.ar/documentos/ensayo-de-largaduracion-de-manejo-deinsumos-en-forma-variable/

8. Bullock, D. G., Bullock, D. S., Nafziger, E. D., Doerge, T. A., Paszkiewicz, S. R., Carter, P. R., \& Peterson, T. A. (1998). Does variable rate seeding of corn pay?. Agronomy Journal, 90(6), 830-836.

9. Cerliani, C., Gomez, M. F., Naville, R., Balboa, G., \& Espósito, G. (2014) densidad de siembra optima de maíz (Zea mays) ajustada por zonas de manejo. X Congreso del maíz HD. Una mirada en alta definición. AIANBA, Buenos Aires, Argentina.

10. D’Andrea, K. E., Otegui, M. E., \& Cirilo, A. G. (2008). Kernel number determination differs among maize hybrids in response to nitrogen. Field Crops Research, 105(3), 228-239.

11. Degioanni, A., De Prada, J.D. \& Cisneros, J.M. (2008). Características del área de estudio, unidades ambientales y productores de la muestra. Capítulo 2. En: Percepción económica y visión de los productores agropecuarios de los problemas ambientales en el Sur de Córdoba, Argentina. Ed. de Prada, J.D. y J.A. Penna. Pp. 23-30.

12. Di Matteo, J. A., Ferreyra, J. M., Cerrudo, A. A., Echarte, L., \& Andrade, F. H. (2016). Yield potential and yield stability of Argentine maize hybrids over 45 years of breeding. Field Crops Research, 197, 107-116.

13. Di Rienzo J.A., Casanoves F., Gonzalez L., Tablada M., Diaz, M.D.P, Robledo C.W. y Balzarini M.G. (2005). Estadisticas para las ciencias 
agropecuarias. Sexta edición. Editorial Brujas. Córdoba, Argentina. $340 \mathrm{pp}$.

14. Di Rienzo J.A., Casanoves F., Balzarini M.G., Gonzalez L., Tablada M., Robledo C.W. (2016) InfoStat versión 2016. Grupo InfoStat, FCA, Universidad Nacional de Córdoba, Argentina. URL http://www.infostat.com.ar

15. Echarte, L., \& Andrade, F. H. (2003). Harvest index stability of Argentinean maize hybrids released between 1965 and 1993. Field Crops Research, 82(1), 1-12.

16. Echarte, L., \& Tollenaar, M. (2006). Kernel set in maize hybrids and their inbred lines exposed to stress. Crop science, 46(2), 870-878.

17. Echarte, L., Nagore, L., Di Matteo, J., Cambareri, M., Robles, M., \& Della Maggiora, A. (2013). Grain yield determination and resource use efficiency in maize hybrids released in different decades. In Agricultural Chemistry. InTech.

18. Echeverría, H. E., \& García, F. O. (2014). Fertilidad de suelos y fertilización de cultivos (No. 631.8). INTA.

19. Edmeades, G. O., \& Daynard, T. B. (1979). The relationship between final yield and photosynthesis at flowering in individual maize plants. Canadian Journal of Plant Science, 59(3), 585-601.

20. Espósito, G. P. (2013). Análisis de la variabilidad espacio-temporal de la respuesta al nitrógeno en maíz mediante un modelo econométrico mixto espacial (MEME). Tesis para optar al grado de Doctor. Universidad Nacional de Córdoba. Argentina.108 p.

21. Hernández, F., Amelong, A., \& Borrás, L. (2014). Genotypic differences among Argentinean maize hybrids in yield response to stand density. Agronomy Journal, 106(6), 2316-2324.

22. Hörbe, T. A. N., Amado, T. J. C., Ferreira, A. D. O., \& Alba, P. J. (2013). Optimization of corn plant population according to management zones in Southern Brazil. Precision Agriculture, 14(4), 450-465.

23. INTA \& Agencia Córdoba Ambiente. 2003. Recursos naturales de la provincia de Córdoba. Los suelos. Nivel de reconocimiento, $\mathrm{E}=$ 1:500.000. Agencia Córdoba D.A.C. y T. S.E.M. Córdoba, Argentina. 567 pp.

24. Kiniry, J. R., Williams, J. R., Vanderlip, R. L., Atwood, J. D., Reicosky, D. C., Mulliken, J., \& Wiebold, W. J. (1997). Evaluation of two maize models for nine US locations. Agronomy Journal, 89(3), 421-426.

25. Lee, E. A., \& Tollenaar, M. (2007). Physiological basis of successful breeding strategies for maize grain yield. Crop Science, 47(Supplement_3), S-202. 
26. Martínez Bologna, G., Castro, S., Cerliani, C., Balboa, G., Naville, R., \& Espósito, G. (2014) densidad de siembra de maíz asociada a la variabilidad espacial del suelo.XXIV Congreso Argentino de la Ciencia del Suelo. Bahía Blanca, Buenos Aires, Argentina.

27. Moral, F. J., Terrón, J. M., \& Da Silva, J. M. (2010). Delineation of management zones using mobile measurements of soil apparent electrical conductivity and multivariate geostatistical techniques. Soil and Tillage Research, 106(2), 335-343.

28. Nagore, M. L., Echarte, L., Andrade, F. H., \& Della Maggiora, A. (2014). Crop evapotranspiration in Argentinean maize hybrids released in different decades. Field Crops Research, 155, 23-29.

29. Ritchie, S. W., Hanway, J. J., \& Benson, G. O. (1982). How a plant crop develops. Spec. Rep, 48.

30. Sarlangue, T., Andrade, F. H., Calviño, P. A., \& Purcell, L. C. (2007). Why do maize hybrids respond differently to variations in plant density? Agronomy Journal, 99(4), 984-991.

31. Schepers, A. R., Shanahan, J. F., Liebig, M. A., Schepers, J. S., Johnson, S. H., \& Luchiari, A. (2004). Appropriateness of management zones for characterizing spatial variability of soil properties and irrigated corn yields across years. Agronomy Journal, 96(1), 195-203.

32. Shanahan, J. F., Doerge, T. A., Johnson, J. J., \& Vigil, M. F. (2004). Feasibility of site-specific management of corn hybrids and plant densities in the Great Plains. Precision Agriculture, 5(3), 207-225.

33. Tollenaar, M., Dwyer, L. M., \& Stewart, D. W. (1992). Ear and kernel formation in maize hybrids representing three decades of grain yield improvement in Ontario. Crop Science, 32(2), 432-438.

34. Tollenaar, M., \& Lee, E. A. (2011). 2 Strategies for Enhancing Grain Yield in Maize. Plant breeding reviews, 34, 37.

35. Vrindts, E., Mouazen, A. M., Reyniers, M., Maertens, K., Maleki, M. R., Ramon, H., \& De Baerdemaeker, J. (2005). Management zones based on correlation between soil compaction, yield and crop data. Biosystems Engineering, 92(4), 419-428. 